\title{
Homogeneous Floquet time crystal protected by gauge invariance
}

\author{
Angelo Russomanno, ${ }^{1,2,3, *}$ Simone Notarnicola, ${ }^{4,5}$ Federica Maria Surace, $, 1,6$ \\ Rosario Fazio, ${ }^{1,7}$ Marcello Dalmonte, ${ }^{1,6}$ and Markus Heyl ${ }^{3}$ \\ ${ }^{1}$ Abdus Salam ICTP, Strada Costiera 11, I-34151 Trieste, Italy \\ ${ }^{2}$ NEST, Scuola Normale Superiore and Istituto Nanoscienze-CNR, I-56126 Pisa, Italy \\ ${ }^{3}$ Max-Planck-Institut für Physik Komplexer Systeme, Nöthnitzer Strasse 38, D-01187 Dresden, Germany \\ ${ }^{4}$ Dipartimento di Fisica e Astronomia “Galileo Galilei”, via Marzolo 8, I-35131 Padova, Italy \\ ${ }^{5}$ INFN, Sezione di Padova, via Marzolo 8, I-35131 Padova, Italy \\ ${ }^{6}$ SISSA, Via Bonomea 265, I-34136 Trieste, Italy \\ ${ }^{7}$ Dipartimento di Fisica, Università di Napoli “Federico II”, Monte S. Angelo, I-80126 Napoli, Italy
}

(Received 18 June 2019; revised manuscript received 28 September 2019; published 6 January 2020)

\begin{abstract}
We show that homogeneous lattice gauge theories can realize nonequilibrium quantum phases with long-range spatiotemporal order protected by gauge invariance instead of disorder. We study a kicked $\mathbb{Z}_{2}$-Higgs gauge theory and find that it breaks the discrete temporal symmetry by a period doubling. In a limit solvable by Jordan-Wigner analysis we extensively study the time-crystal properties for large systems and further find that the spatiotemporal order is robust under the addition of a solvability-breaking perturbation preserving the $\mathbb{Z}_{2}$ gauge symmetry. The protecting mechanism for the nonequilibrium order relies on the Hilbert space structure of lattice gauge theories, so that our results can be directly extended to other models with discrete gauge symmetries.
\end{abstract}

DOI: 10.1103/PhysRevResearch.2.012003

Introduction. Isolated quantum matter can feature phases with long-range order in highly excited states that cannot be captured by thermodynamic ensembles $[1,2]$. This crucially relies on ergodicity breaking and a failure of the Eigenstate Thermalization Hypothesis [3]. One robust mechanism for achieving such nonergodic behavior is to impose strong disorder giving rise to the many-body localized (MBL) phase [2,4-8], which can host long-range ordered phases such as the MBL-spin glass [1,9] or Floquet time crystals [10-16]. Recently, it has been realized that lattice gauge theories (LGTs) entail another robust mechanism for nonergodic dynamics in short-ranged systems protected by gauge invariance instead of disorder [6-8] due to the specific structure of their Hilbert spaces, which are built up of disconnected superselection sectors [7]. However, it has remained an open question to what extent they can also accommodate nonequilibrium phases with long-range order and therefore to what extent they can contribute to the open quest of realizing robust nonequilibrium ordered phases of homogeneous quantum many-body systems.

In this work we introduce a phase of quantum matter unique to LGTs that exhibits both spatial and temporal order thereby constituting a genuine nonequilibrium phenomenon. In particular, we show that homogeneous LGTs can feature robust time-crystalline phases in short-range systems protected by gauge invariance as opposed to previously studied

\footnotetext{
*angelo.russomanno@tiscali.it

Published by the American Physical Society under the terms of the Creative Commons Attribution 4.0 International license. Further distribution of this work must maintain attribution to the author(s) and the published article's title, journal citation, and DOI.
}

cases that were relying on the presence of strong disorder. In order to realize such a "gauge time crystal," we introduce a periodically kicked $\mathbb{Z}_{2}$ LGT which, as we find, displays a subharmonic response to the external drive associated with a period doubling (see Fig. 1). We identify two necessary properties essential to realize a Floquet time crystal within the considered scheme: (i) in a given superselection sector the LGT has to realize bond instead of field disorder in contrast to previously studied models of disorder-free localization [6-8]; (ii) the gauge symmetry has to be discrete and different from many previously studied nonergodic U(1) LGTs [6,7]. We solve the considered kicked $\mathbb{Z}_{2}$ LGT exactly by a mapping onto a free fermionic theory using a Jordan-Wigner (J-W) transformation, which allows us to explore the phase diagram for large system sizes. We observe that the Floquet states appear in pairs with a quasienergy difference of $\pi$, so that our system shares many of the features of the $\pi$-spin glass in a periodically kicked Ising chain with quenched disorder [10]. Importantly, we find that this gauge time crystal represents a robust phase which does not require fine-tuning and persists over a wide range of parameters. In particular, we also study the influence of perturbations breaking the exact solvability and preserving the $\mathbb{Z}_{2}$ gauge symmetry, where we find numerical evidence for stability by means of exact diagonalization. We discuss how to extend our analysis to a $\mathbb{Z}_{N}$-symmetric LGT along the lines of [17]. The mechanism behind this time-crystalline phase relies on gauge invariance and can therefore be directly extended to other LGTs with discrete gauge symmetries. Importantly, our observation of a robust time-crystalline phase in a homogeneous shortranged system goes beyond recent approaches which lead to prethermal spatiotemporal order [18-22], or require longrange interactions in driven unitary $[23,24]$ and dissipative dynamics [25-32]. 
(a)
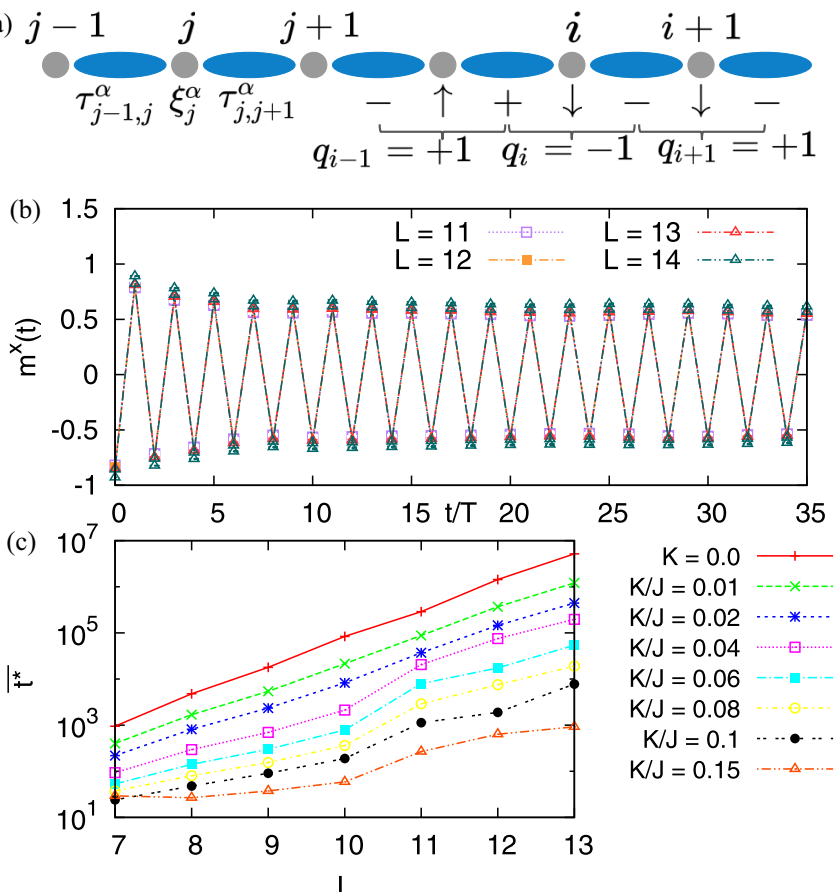

FIG. 1. (a) Schematic illustration of the $\mathbb{Z}_{2}$ Higgs-LGT, with matter fields on the lattice sites $l$, represented by Pauli operators $\hat{\xi}_{j}^{\alpha}$, and gauge degrees of freedom by $\hat{\tau}_{j, j+1}^{\alpha}$ on the links. The local gauge symmetry imposes a locally conserved quantity given by the eigenvalues $q_{i}$ of the operator $\hat{G}_{i}=-\hat{\tau}_{i-1, i}^{x} \hat{\xi}_{i}^{z} \hat{\tau}_{i, i+1}^{x}$, which are included for a simple example, where $\uparrow \downarrow$ represents the eigenvalues of $\xi_{j}^{z}$ and \pm of $\tau_{j, j+1}^{x}$, respectively. (b) Stroboscopic dynamics of the magnetization $m^{x}(t)$ of the gauge degrees of freedom in the kicked $\mathbb{Z}_{2}$ LGTs displaying period-doubling oscillations. (c) The decay time $\overline{t^{*}}$ of the period-doubling oscillations increases exponentially with system size $L$ marking the presence of a time-crystal behavior. We have taken a $\mathbb{Z}_{2}$-symmetry-breaking initial state with $f=0.8$ [see discussion after Eq. (7)]. Numerical parameters: $\phi=1.02 \pi$, $h / J=0.2, m / J=0.5, J T=1.0, N_{\text {real }}=48$, and $K / J=0.1$ in (b).

The model. We consider a $\mathbb{Z}_{2}$ Higgs-LGT in one spatial dimension. The theory describes the dynamics of Higgs fields, defined by Pauli-matrix operators $\hat{\xi}_{j}^{\alpha}$ at vertex $j$ on the lattice, coupled to $\mathbb{Z}_{2}$ gauge fields, defined by $\mathbb{Z}_{2}$ parallel transporters $\hat{\tau}_{j, j+1}^{x}$ at the bond $(j, j+1)$ as illustrated in Fig. 1(a). The system Hamiltonian reads $[33,34]$

$$
\hat{H}_{0}=\frac{m}{2} \sum_{j=1}^{L} \hat{\xi}_{j}^{z}+J \sum_{j=2}^{L-1} \hat{\tau}_{j-1, j}^{x} \hat{\tau}_{j, j+1}^{x}+h \sum_{j=1}^{L-1} \hat{\xi}_{j}^{x} \hat{\tau}_{j, j+1}^{z} \hat{\xi}_{j+1}^{x} .
$$

The Higgs-field operators can also be interpreted as hard-core bosons $\hat{b}_{j}$ with $\hat{\xi}_{j}^{x}=\hat{b}_{j}^{\dagger}+\hat{b}_{j}$. The first two terms denote mass and gauge interactions, while the third describes the coupling between the Higgs and gauge fields. We drive the $\mathbb{Z}_{2}$ HiggsLGT out of equilibrium by periodically kicking the strength of the Higgs-gauge coupling, leading to the following timedependent Hamiltonian

$$
\hat{H}(t)=\hat{H}_{0}+\frac{\phi}{2} \sum_{n=-\infty}^{+\infty} \delta(t-n T) \sum_{j=1}^{L-1} \hat{\xi}_{j}^{x} \hat{\tau}_{j, j+1}^{z} \hat{\xi}_{j+1}^{x} .
$$

This system exhibits a local symmetry: $\hat{H}(t)$ commutes with the operators $\hat{G}_{j}=-\hat{\tau}_{j-1, j}^{x} \hat{\tau}_{j, j+1}^{x} \hat{\xi}_{j}^{z}$ (which can be understood as the complex exponentials of the local Gauss' operators). Thus, the Hilbert space of size $2^{2 L-1}$ is partitioned in $\mathcal{N}=2^{L}$ superselection sectors, where all the states $\left|\Psi_{\left\{q_{\alpha}\right\}}\right\rangle$ in a given sector are identified by the same set of local static charges $q_{j}= \pm 1$ via $\hat{G}_{j}\left|\Psi_{\left\{q_{\alpha}\right\}}\right\rangle=q_{j}\left|\Psi_{\left\{q_{\alpha}\right\}}\right\rangle$.

In the following we consider initial product states of the form $|\Psi\rangle=|\varphi\rangle_{H} \otimes|\psi\rangle_{g}$ where $|\varphi\rangle_{H}$ is a product state which satisfies ${ }_{H}\left\langle\varphi\left|\hat{\xi}_{j}^{z}\right| \varphi\right\rangle_{H}=0$ for all $j=1, \ldots, N$ and $|\psi\rangle_{g}$ is the initial condition for the gauge degrees of freedom, which we will specify later in the text. Such initial conditions, which represent superpositions over many superselection sectors, can yield robust nonergodic behavior for LGTs and disorderfree localization [6-8,35]. Concretely, for our $\mathbb{Z}_{2}$ LGT the dynamics in a given superselection sector specified by the charges $\left\{q_{\alpha}\right\}$ is determined by an effective Hamiltonian [36]

$$
\hat{H}_{\left\{q_{\alpha}\right\}}(t)=\sum_{j=2}^{L-1} J_{j} \hat{\tau}_{j-1, j}^{x} \hat{\tau}_{j, j+1}^{x}+h(t) \sum_{j=1}^{L-1} \hat{\tau}_{j, j+1}^{z},
$$

with $\quad h(t)=h+(\phi / 2) \sum_{n=-\infty}^{+\infty} \delta(t-n T), \quad J_{j}=[1-$ $\left.q_{j} m /(2 J)\right]$, and the $\hat{\tau}_{j, j+1}^{\alpha}$ operators redefined with respect to Eqs. (1) and (2) (see [36] for details). This integration is related to the duality between Ising models and Ising LGTs [37,38]. As a result the Hamiltonian becomes a kicked transverse-field Ising chain with binary bond disorder due to $q_{j}= \pm 1$, which can be solved exactly via a J-W transformation for large systems. We emphasize that, due to the presence of degeneracies in the unperturbed Floquet spectrum, it is a priori less clear whether bond disorder, with respect to one with a continuous distribution, is able to induce MBL in order to get a time crystal. We will also study the influence of a perturbation of the form $\hat{H}_{K}=$ $4 K \sum_{j=2}^{L-1} \hat{\xi}_{j-1}^{x} \hat{\tau}_{j-1, j}^{z} \hat{\tau}_{j, j+1}^{z} \hat{\xi}_{j+1}^{x}$ breaking the J-W solvability. After the integration it adds a transverse interaction term for the gauge fields,

$$
\hat{H}_{\left\{q_{\alpha}\right\}}^{K}(t)=\hat{H}_{\left\{q_{\alpha}\right\}}(t)+4 K \sum_{j=2}^{L-1} \hat{\tau}_{j-1, j}^{z} \hat{\tau}_{j, j+1}^{z} .
$$

We solve the dynamics of the LGT in a set of $N_{\text {real }}$ randomly chosen superselection sectors and finally perform an average when computing observables. In the shown data we include error bars resulting from the finiteness of $N_{\text {real }}$. But let us emphasize again that the overall problem is homogeneous both in the initial condition and in the Hamiltonian.

Initial conditions and observables. In order to reveal both the temporal and spatial order we use two complementary setups.

On the one hand, we take initial conditions which explicitly break the $\mathbb{Z}_{2}$ symmetry of the model yielding a nonzero magnetization $m^{x}$ for the gauge degrees of freedom which we then monitor in the subsequent evolution:

$$
m^{x}(t)=\frac{1}{L-1} \sum_{j=1}^{L-1}\left\langle\hat{\tau}_{j, j+1}^{x}\right\rangle_{t},
$$

where we have defined $\langle\cdots\rangle_{t} \equiv \overline{g\langle\psi(t)|\cdots| \psi(t)\rangle_{g}}$ and the overline marks the average over the $N_{\text {real }}$ pseudodisorder 
realizations [39]. In this way we obtain direct access to the time-crystalline period-doubling dynamics. In Fig. 1(b) we show results for $m^{x}(t)$ in the fully interacting case $K \neq 0$ obtained through exact diagonalization. We see the existence of period-doubling oscillations which are persistent for an infinite time in the thermodynamic limit. We show this fundamental property of persistence [11] in Fig. 1(c), where we see that the decay time $\overline{t^{*}}$ of the period-doubling oscillations exponentially scales to infinity with the system size. We determine $\overline{t^{*}}$ as the time after which $(-1)^{t / T} m^{x}(t)$ changes sign $[17,40]$ averaged over disorder.

On the other hand, we can choose initial conditions which are $\mathbb{Z}_{2}$ symmetric with a vanishing magnetization $m^{x}(t)$, which allows us to address the spatial long-range ordering in the system. For that purpose we study the correlation parameter

$$
\mathcal{S}_{t}^{x x}=\frac{1}{(L-1)(L-2)} \sum_{i, j=1,(i \neq j)}^{L-1}\left\langle\hat{\tau}_{j, j+1}^{x} \hat{\tau}_{i, i+1}^{x}\right\rangle_{t},
$$

with $\langle\cdots\rangle_{t}$ defined as above. Whenever $\mathcal{S}_{t}^{x x}>0$ while at the same time $m^{x}(t)=0$, the system exhibits long-range spatial order.

Exactly solvable case. Let us first focus on the case with $K=0$, where the model can be mapped onto a system of noninteracting fermions by means of a $\mathrm{J}-\mathrm{W}$ transformation. In each superselection sector $\left\{q_{\alpha}\right\}$ we initialize the dynamics with the same initial state $|\psi\rangle_{g}$ chosen as the ground state of the Hamiltonian $\hat{H}_{0}=\sum_{j=2}^{L-1} \hat{\tau}_{j-1, j}^{x} \hat{\tau}_{j, j+1}^{x}+h_{0} \sum_{j=1}^{L-1} \hat{\tau}_{j, j+1}^{z}$. This state has a nonvanishing correlation parameter if $h_{0}<1$ and is symmetric under $\mathbb{Z}_{2}$ which allows us to address the long-range spatial ordering in the system; for a study of the temporal order we perform a spectral analysis, as we are going to detail below. In the J-W framework it is well known how to numerically study the dynamics and how to evaluate the correlation parameter as a Pfaffian (see [41-45]). Here it is enough to say that the dynamics is induced by an effective $2(L-1) \times 2(L-1)$ time-periodic single-particle Hamiltonian. This is important to mention because we can compute the $2(L-1)$ single-particle Floquet states and the $2(L-1)$ single-particle quasienergies $\epsilon_{\alpha}$ (see, for instance, [46]). These quantities will play an important role in what follows.

We find that the correlation order parameter reaches an asymptotic value $\mathcal{S}_{\text {asy }}^{x x}$ after a transient [see the discussion below Eq. (7)]. We plot the long-time value of $\mathcal{S}_{t}^{x x}$ as a function of kicking strength $\phi$ for different values of $L$ in the main panel of Fig. 2. We observe three regimes whose separating phase boundaries we indicate by the colored zones. In regimes (i) and (iii) $\mathcal{S}_{\text {asy }}^{x x}$ converges to a nonzero value as $L \rightarrow \infty$, while in regime (ii) $\mathcal{S}_{\text {asy }}^{x x}$ vanishes as the $L$ is increased (see also the inset of Fig. 2). Both regions (i) and (iii) mark the existence of an eigenstate phase [1,2], where eigenstates exhibit long-range spatial order (as in [9], for instance). This eigenstate order is protected by disorder and MBL since in a clean short-range one-dimensional spin interacting thermalizing system with $\mathbb{Z}_{2}$ symmetry such order is impossible (this result is easily shown for clean $\mathbb{Z}_{2}$ one-dimensional spin chains [47], where long-range order is possible only in the ground state).

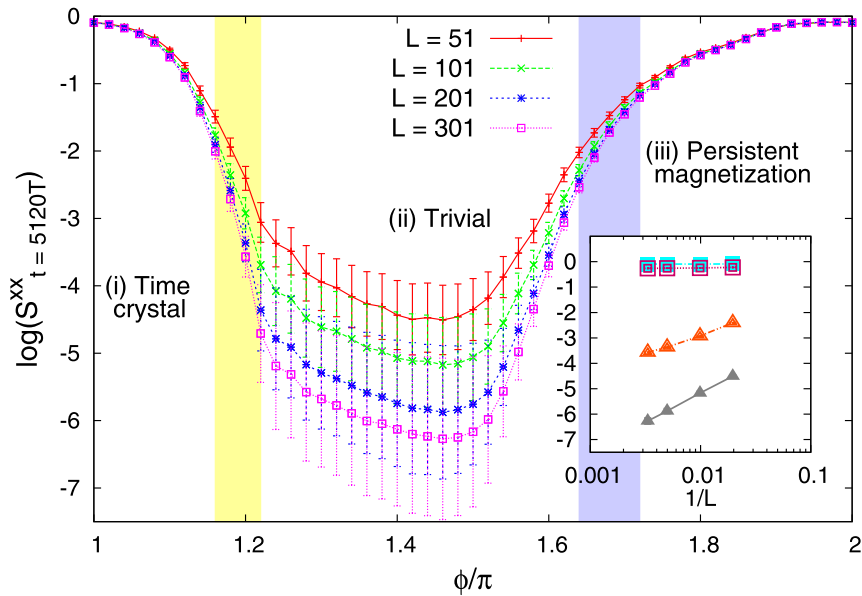

FIG. 2. Spatial long-range order in the exactly solvable limit $K=0$ : Asymptotic long-time value $\mathcal{S}_{\text {asy }}^{x x}$ versus $\phi$ for different values of system size $L$. Inset: System-size dependence of $\mathcal{S}_{\text {asy }}^{x x}$ from top to bottom for $\phi / \pi=1,1.06,1.2,1.4$. Numerical parameters as in Fig. 1 , except $K=0$ and $N_{\text {real }} \geqslant 104$.

Although the behavior of $\mathcal{S}_{\text {asy }}^{x x}$ is qualitatively similar in both (i) and (iii), these two regions mark different phases since (i) in addition also supports temporal order. An example of this property for $\phi=1.02 \pi$ can be seen in Fig. 1(c) (curve with $K=0$ ): The system is initialized in a state explicitly breaking the $\mathbb{Z}_{2}$ symmetry and the decay time $\overline{t^{*}}$ exponentially increases with the system size. This fact can be understood by an analysis of the Floquet spectrum [10]. The presence of a temporal time-crystal ordering corresponds to spectral pairing, where each Floquet state has a partner with quasienergy shifted by $\pi$. This situation is realized if there is a single-particle quasienergy exactly at $\pi$ with a marked gap separating it from the rest of the spectrum. In this way it does not hybridize with the bulk, and each many-body Floquet state has a $\pi$-shifted partner obtained by adding the quasiparticle with quasienergy $\pi$. We evaluate this gap as $\overline{\delta_{\pi}}=$ $\frac{1}{N_{\text {real }}} \sum_{q=1}^{N_{\text {real }}}\left[\epsilon_{2 L-2}^{(q)}-\epsilon_{2 L-3}^{(q)}\right]$ [48] and plot it in Fig. 3. We see that it is nonvanishing in all of regime (i). Moreover, as we show in [36], in this regime $\epsilon_{2 L-2}$ averaged over the disorder is exactly equal to $\pi$. In [36] we show also that the single-particle bulk Floquet states are always Anderson localized. This is very important, because without localization it is possible to have

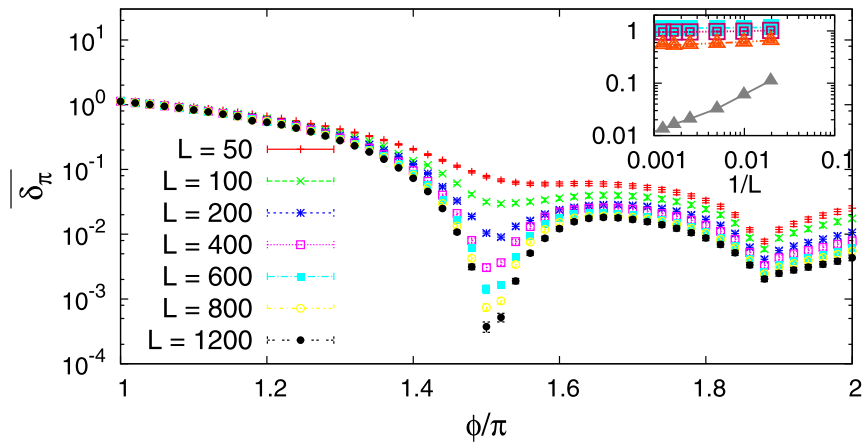

FIG. 3. Disorder-averaged single-particle Floquet spectral gap $\overline{\delta_{\pi}}$ as a function of $\phi$ for different $L$ using the same parameters as in Fig. 2. Inset: System-size dependence of $\overline{\delta_{\pi}}$. 
a gap in the Floquet spectrum at $\pi$ and still observe no time crystal (see, for instance, [46]): In the absence of localization, local operators expand in time obeying the Lieb-Robinson bound and no time-periodic behavior whatsoever is possible [49]. Of course, the transition to localization and the one to glassy order of the excited eigenstates are independent [9], and this is the reason why the transition from regime (i) and (ii) occurs at a value of $\phi$ different from the one where $\overline{\delta_{\pi}}$ vanishes. In Fig. 2 we have initialized with a specific value of $h_{0}$, but we have checked that the presented phenomenology does not depend on this choice.

General case. At this point we break $\mathrm{J}-\mathrm{W}$ solvability by considering the term of Eq. (4), with $K \neq 0$. We consider a value of $\phi$ for which we see this phenomenon at $K=0$; then we take $K \neq 0$ and we study the properties of the asymptotic correlation parameter. An interval of $K$ where this quantity does not scale with the size would mark the persistence of the time crystal. We now perform a conventional exactdiagonalization simulation of the system, up to size $L=13$. To evaluate the asymptotic correlation parameter, we can resort to the Floquet diagonal-ensemble average and we get

$$
\mathcal{S}_{\text {asy }}^{x x}=\sum_{i, j=1,(i \neq j)}^{L-1} \sum_{\beta=1}^{\mathcal{N}} \frac{\overline{\left|R_{\beta}\right|^{2}{ }_{g}\left\langle\phi_{\beta}\left|\hat{\tau}_{j, j+1}^{x} \hat{\tau}_{i, i+1}^{x}\right| \phi_{\beta}\right\rangle_{g}}}{(L-1)(L-2)},
$$

where $\left|\phi_{\beta}\right\rangle_{g}$ are the many-body Floquet states, $\mathcal{N}$ is the dimension of the Hilbert space, and $R_{\beta} \equiv{ }_{g}\left\langle\psi(0) \mid \phi_{\beta}\right\rangle_{g}$ denotes the overlap with the initial state. We remark that we can use Eq. (7) even if the many-body Floquet quasienergies $\mu_{\beta}$ appear in degenerate pairs, due to the $\mathbb{Z}_{2}$ symmetry. The point is that the operators $\hat{\tau}_{j, j+1}^{x} \hat{\tau}_{i, i+1}^{x}$ commute with the same $\mathbb{Z}_{2}$ symmetry and hence have no matrix elements between states with different parity (see the detailed demonstration along the lines of [50] in [36]). We plot the dependence of $\mathcal{S}_{\text {asy }}^{x x}$ versus $K$ for different $L$ in Fig. 4. We take two different initial conditions: in the upper panel we take the state with all the spins pointing down along the $x$ axis $\left(|\psi\rangle_{g}=\left|s_{1,2}^{x}=-1 \ldots s_{j, j+1}^{x}=-1 \ldots s_{L-1, L}^{x}=-1\right\rangle_{g}\right) ; \quad$ in

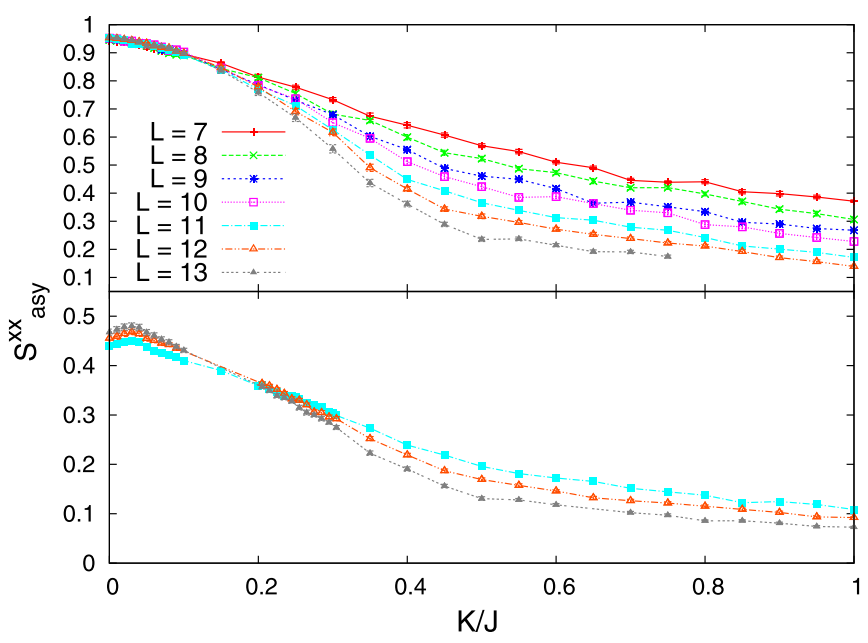

FIG. 4. Stability of the time crystal: $\mathcal{S}_{\text {asy }}^{x x}$ as a function of $K$ for various $L$ and two different initial states with $f=1$ (upper panel) and $f=0.8$ (lower panel). Numerical parameters as in Fig. 1 . the lower panel we take the uniform superposition of all the eigenstates of $\hat{\tau}_{j, j+1}^{x} \forall j$ obeying the condition $\sum_{j=1}^{L-1} s_{j, j+1}^{x} \leqslant-(L-1) f$ with $f=0.8$. We see that for $K \lesssim 0.2$ there is no decrease with $L$, marking the persistence of the time-crystal behavior. This persistence can be seen also in Fig. 1(c) where the $\overline{t^{*}}$ introduced above exponentially increases with $L$.

Time crystallinity in Abelian lattice gauge theories. We now investigate more generally if time crystallinity can appear in disorder-free Abelian LGTs in $(1+1)$ dimension. We consider the generic Hamiltonian coupling Higgs fields to Abelian gauge fields [51]:

$$
\begin{aligned}
\hat{H}= & m \sum_{j=1}^{L}(-1)^{j} \hat{n}_{j}+\sum_{j=1}^{L-1}\left(\hat{\varphi}_{j}^{\dagger} \hat{\mathcal{U}}_{j, j+1} \hat{\varphi}_{j+1}+\text { H.c. }\right) \\
& +\frac{g^{2}}{2} \sum_{j=1}^{L-1} \hat{E}_{j, j+1}^{2}+\hat{H}(t),
\end{aligned}
$$

where $\hat{n}_{j}=\hat{\varphi}_{j}^{\dagger} \hat{\varphi}_{j}$ is the Higgs occupation on site $j$ and $\hat{E}_{j, j+1}, \hat{\mathcal{U}}_{j, j+1}$ are, respectively, the electric field and the parallel transporter, and $\hat{H}(t)$ is defined analogously to the $\mathbb{Z}_{2}$ case above. The electric-field interaction energy is local in these theories, differently from the $\mathbb{Z}_{2}$ term involving at least two neighboring sites. For a $\mathbb{Z}_{N}$ LGT (i.e., a theory where now $\hat{\mathcal{U}}_{j, j+1}$ and $\hat{E}_{j, j+1}$ are not Pauli matrices but the more general clock operators), we can use a similar approach as the one used in the $\mathbb{Z}_{2}$ LGT. We consider an initial state where matter is in an equal-weight superposition of all possible eigenvalues of the Higgs number operator, and the gauge fields are in a generic state. The evolution of such states can be mapped exactly into the one of $\mathbb{Z}_{N}$ clock models under the effect of quasirandom local fields: since the latter class of models has been shown to display time-crystal behavior for small values of $N$ and random disorder [17], it is natural to expect that the mechanism discussed above holds true also for $N>2$. This mechanism does not work for continuous U(1) LGTs (see [36] for details), which, however, does not exclude other ones for the generation of time crystals in such theories.

Concluding discussion. In this work we have demonstrated that homogeneous LGTs can realize time-crystal phases, where the protecting nonergodicity is enforced by the local constraints imposed by gauge invariance. In more general terms, our results show that homogeneous LGTs can realize eigenstate order, which naturally leads to the question to what extent also other eigenstate phases can occur in homogeneous LGTs, e.g., analogs of the MBL-spin glass [1,9] or topological order at elevated energy densities [52]. Our results are of immediate relevance to experiments realizing lattice gauge theory dynamics [53-55] in both trapped ions [56] and cold atom systems [57,58]. In particular, scalable proposals have been formulated [59,60], and several experiments have already demonstrated the building blocks [61-64] for discrete lattice gauge theories of relevance to gauge time crystals.

Further, our results can be directly extended to $\mathbb{Z}_{N}$ LGTs which opens up the possibility, in principle, of generating period $N$-tupling time crystals. While our approach cannot be immediately applied to LGTs with continuous groups, 
it would be intriguing to see whether discrete non-Abelian symmetries can also support the formation of defect-free time crystals.

Acknowledgments. We acknowledge fruitful discussions with R. Khasseh and M. Wauters. A.R. and R.F. thank the European Union for partial financial support through QUIC project (under Grant Agreement No. 641122). A.R. thanks the Max-Planck-Institut für Physik Komplexer Systeme for par- tial financial support and the warm hospitality received during the preparation of this work. S.N. and M.D. acknowledge partial support by the H2020 Project-QUANTUM FLAGSHIPPASQUANS (2019-2022). We are indebted to G. E. Santoro for the subroutine performing the diagonalization of unitary operators. This work is partly supported by the ERC under Grant No. 758329 (AGEnTh) and by the QUANTERA project QTFLAG.
[1] D. A. Huse, R. Nandkishore, V. Oganesyan, A. Pal, and S. L. Sondhi, Phys. Rev. B 88, 014206 (2013).

[2] R. Nandkishore and D. A. Huse, Annu. Rev. Condens. Matter Phys. 6, 15 (2015).

[3] L. D'Alessio, Y. Kafri, A. Polkovnikov, and M. Rigol, Adv. Phys. 65, 239 (2016).

[4] E. Altman and R. Vosk, Annu. Rev. Condens. Matter Phys. 6, 383 (2015).

[5] D. A. Abanin, E. Altman, I. Bloch, and M. Serbyn, Rev. Mod. Phys. 91, 021001 (2019).

[6] A. Smith, J. Knolle, D. L. Kovrizhin, and R. Moessner, Phys. Rev. Lett. 118, 266601 (2017).

[7] M. Brenes, M. Dalmonte, M. Heyl, and A. Scardicchio, Phys. Rev. Lett. 120, 030601 (2018).

[8] A. Smith, J. Knolle, R. Moessner, and D. L. Kovrizhin, Phys. Rev. B 97, 245137 (2018).

[9] J. A. Kjäll, J. H. Bardarson, and F. Pollmann, Phys. Rev. Lett. 113, 107204 (2014).

[10] V. Khemani, A. Lazarides, R. Moessner, and S. L. Sondhi, Phys. Rev. Lett. 116, 250401 (2016).

[11] D. V. Else, B. Bauer, and C. Nayak, Phys. Rev. Lett. 117, 090402 (2016).

[12] J. Zhang, P. W. Hess, A. Kyprianidis, P. Becker, A. Lee, J. Smith, G. Pagano, I.-D. Potirniche, A. C. Potter, A. Vishwanath, N. Y. Yao, and C. Monroe, Nature (London) 543, 217 (2017).

[13] S. Pal, N. Nishad, T. S. Mahesh, and G. J. Sreejith, Phys. Rev. Lett. 120, 180602 (2018).

[14] N. Y. Yao, A. C. Potter, I.-D. Potirniche, and A. Vishwanath, Phys. Rev. Lett. 118, 030401 (2017).

[15] J. Rovny, R. L. Blum, and S. E. Barrett, Phys. Rev. Lett. 120, 180603 (2018).

[16] J. Rovny, R. L. Blum, and S. E. Barrett, Phys. Rev. B 97, 184301 (2018).

[17] F. M. Surace, A. Russomanno, M. Dalmonte, A. Silva, R. Fazio, and F. Iemini, Phys. Rev. B 99, 104303 (2019).

[18] D. V. Else, B. Bauer, and C. Nayak, Phys. Rev. X 7, 011026 (2017).

[19] W. C. Yu, J. Tangpanitanon, A. W. Glaetzle, D. Jaksch, and D. G. Angelakis, Phys. Rev. A 99, 033618 (2019).

[20] R. Schäfer, G. S. Uhrig, and J. Stolze, Phys. Rev. B 100, 184301 (2019).

[21] J. Choi et al., Phys. Rev. Lett. 122, 043603 (2019).

[22] T. Iadecola, M. Schecter, and S. Xu, Phys. Rev. B 100, 184312 (2019).

[23] K. Sacha, Phys. Rev. A 91, 033617 (2015).

[24] A. Russomanno, F. Iemini, M. Dalmonte, and R. Fazio, Phys. Rev. B 95, 214307 (2017).
[25] B. Zhu, J. Marino, N. Y. Yao, M. D. Lukin, and E. A. Demler, New J. Phys. 21, 073028 (2019).

[26] K. Tucker, B. Zhu, R. J. Lewis-Swan, J. Marino, F. Jimenez, J. G. Restrepo, and A. M. Rey, New J. Phys. 20, 123003 (2018).

[27] F. Iemini, A. Russomanno, J. Keeling, M. Schirò, M. Dalmonte, and R. Fazio, Phys. Rev. Lett. 121, 035301 (2018).

[28] F. M. Gambetta, F. Carollo, M. Marcuzzi, J. P. Garrahan, and I. Lesanovsky, Phys. Rev. Lett. 122, 015701 (2019).

[29] R. R. W. Wang, B. Xing, G. G. Carlo, and D. Poletti, Phys. Rev. E 97, 020202(R) (2018).

[30] Z. Gong, R. Hamazaki, and M. Ueda, Phys. Rev. Lett. 120, 040404 (2018).

[31] J. O'Sullivan, O. Lunt, C. W. Zollitsch, M. L. W. Thewalt, J. J. L. Morton, and A. Pal, arXiv:1807.09884.

[32] A. Lazarides, S. Roy, F. Piazza, and R. Moessner, arXiv:1904.04820.

[33] M. Creutz, Quarks, Gluons and Lattices (Cambridge University Press, Cambridge, 1997).

[34] E. Fradkin, Field Theories of Condensed Matter Physics, 2nd ed. (Cambridge University Press, Cambridge, 2013).

[35] A. Smith, J. Knolle, R. Moessner, and D. L. Kovrizhin, Phys. Rev. Lett. 119, 176601 (2017).

[36] See Supplemental Material at http://link.aps.org/supplemental/ 10.1103/PhysRevResearch.2.012003 for details on the derivation of the effective Hamiltonian Eq. (3), localization properties of the single-particle Floquet spectrum, demonstration of the convergence of the correlation parameter to the diagonalensemble expression Eq. (7) and explanation for the impossibility of extending this Floquet time-crystal scheme to continuous gauge symmetries.

[37] F. Wegner, J. Math. Phys. 12, 2259 (1971).

[38] B. M. McCoy and M.-L. Yan, Nucl. Phys. B 215, 278 (1983).

[39] The system naturally averages over the pseudodisorder realizations, so the error bars we consider are the fluctuations of the average. They are evaluated as the standard deviation over pseudodisorder of the considered quantity divided by the square root of the number of realizations.

[40] Supplemental Material of Ref. [11].

[41] E. Lieb, T. Schultz, and D. Mattis, Ann. Phys. (NY) 16, 407 (1961).

[42] P. Pfeuty, Ann. Phys. (NY) 57, 79 (1970).

[43] A. Russomanno, A. Silva, and G. E. Santoro, J. Stat. Mech. (2013) P09012.

[44] T. Caneva, R. Fazio, and G. E. Santoro, Phys. Rev. B 76, 144427 (2007). 
[45] E. Barouch and B. M. McCoy, Phys. Rev. A 3, 786 (1971).

[46] A. Russomanno, B.-e. Friedman, and E. G. Dalla Torre, Phys. Rev. B 96, 045422 (2017).

[47] G. G. Batrouni and R. T. Scalettar, Quantum Phase Transitions, in Ultracold Gases and Quantum Information, Lecture Notes of the Les Houches Summer School in Singapore Vol. 91 (Oxford University Press, New York, 2011).

[48] The quasienergies are in increasing order. If there is a spectral gap, $\epsilon_{2 L-2}$ is the one at $\pi$. In this case, due to the particle-hole symmetry, $\epsilon_{1}=-\pi$.

[49] V. Khemani, C. W. von Keyserlingk, and S. L. Sondhi, Phys. Rev. B 96, 115127 (2017).

[50] Angelo Russomanno, Alessandro Silva, and Giuseppe E. Santoro, Phys. Rev. Lett. 109, 257201 (2012).

[51] J. Kogut and L. Susskind, Phys. Rev. D 11, 395 (1975).

[52] Y. Bahri, R. Vosk, E. Altman, and A. Vishwanath, Nat. Commun. 6, 7341 (2015).

[53] U. J. Wiese, Ann. Phys. (NY) 525, 777 (2013).

[54] M. Dalmonte and S. Montangero, Contemp. Phys. 57, 388 (2016).

[55] E. Zohar, I. Cirac, and B. Reznik, Rep. Prog. Phys. 79, 014401 (2016).
[56] E. A. Martinez, C. A. Muschik, P. Schindler, D. Nigg, A. Erhard, M. Heyl, P. Hauke, M. Dalmonte, T. Monz, P. Zoller, and R. Blatt, Nature (London) 534, 516 (2016).

[57] H. Bernien et al., Nature (London) 551, 579 (2017).

[58] F. M. Surace, P. P. Mazza, G. Giudici, A. Lerose, A. Gambassi, and M. Dalmonte, arXiv:1902.09551.

[59] E. Zohar, A. Farace, B. Reznik, and J. I. Cirac, Phys. Rev. Lett. 118, 070501 (2017).

[60] S. Notarnicola, M. Collura, and S. Montangero, arXiv:1907.12579.

[61] M. Anderlini, P. J. Lee, B. L. Brown, J. Sebby-Strabley, W. D. Phillips, and J. V. Porto, Nature (London) 448, 452 (2007).

[62] S. Trotzky, P. Cheinet, S. Fölling, M. Feld, U. Schnorrberger, A. M. Rey, A. Polkovnikov, E. A. Demler, M. D. Lukin, and I. Bloch, Science 319, 295 (2008).

[63] C. Schweizer, F. Grusdt, M. Berngruber, L. Barbiero, E. Demler, N. Goldman, I. Bloch, and M. Aidelsburger, Nat. Phys. 15, 1168 (2019).

[64] A. Mil, T. V. Zache, A. Hegde, A. Xia, R. P. Bhatt, M. K. Oberthaler, P. Hauke, J. Berges, and F. Jendrzejewski, arXiv:1909.07641. 